\title{
First Simulation Results for the Photon in a Non-Commutative Space
}

\author{
W. Bietenholz ${ }^{a}$, F. Hofheinz ${ }^{a}$, J. Nishimura ${ }^{b}$, Y. Susaki ${ }^{b}{ }^{c}$ and J. Volkholz ${ }^{a}$ \\ anstitut für Physik, Humboldt Universität zu Berlin, Newtonstr. 15, 12489 Berlin, Germany

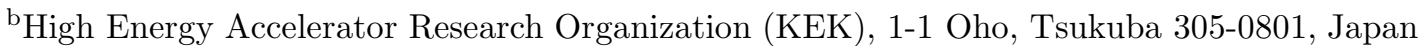 \\ cInstitute of Physics, University of Tsukuba, Tsukuba, Ibaraki 305-8571, Japan
}

We present preliminary simulation results for QED in a non-commutative 4d space-time, which is discretized to a fuzzy lattice. Its numerical treatment becomes feasible after its mapping onto a dimensionally reduced twisted Eguchi-Kawai matrix model. In this formulation we investigate the Wilson loops and in particular the Creutz ratios. This is an ongoing project which aims at non-perturbative predictions for the photon, which can be confronted with phenomenology in order to verify the possible existence of non-commutativity in nature.

\section{THE NON-COMMUTATIVE U(1) MODEL}

We investigate non-perturbatively QED on a non-commutative (NC) space-time [1, where a commutation relation of the form

$$
\left[\hat{x}_{\mu}, \hat{x}_{\nu}\right]=i \Theta_{\mu \nu}
$$

is introduced. This has the effect of replacing points in regular space with "Planck cells". $\Theta_{\mu \nu}$ is a real and antisymmetric non-commutativity tensor.

Here we address pure $\mathrm{U}(1)$ gauge theory in a Euclidean NC space-time. Our main tool is the star product, which is defined as

$f(x) \star g(x)=f(x) \exp \left(\frac{i}{2} \overleftarrow{\partial_{\mu}} \Theta_{\mu \nu} \overrightarrow{\partial_{\nu}}\right) g(x)$.

This definition absorbs the "space deformation" into the products of fields, thereby allowing us to treat the space-time operators $\hat{x}$ and functions thereof as regular space-time coordinates again,

$\hat{\phi}(\hat{x}) \hat{\psi}(\hat{x}) \rightarrow \phi(x) \star \psi(x)$.

Equipped with this tool we are able to write down the gauge action of the pure $\mathrm{U}(1)$ theory as

$S[A]=-\frac{1}{4 g^{2}} \int \mathrm{d}^{4} x \operatorname{Tr}\left[F_{\mu \nu}(x) \star F_{\mu \nu}(x)\right]$,

where $F_{\mu \nu}$ is given by

$F_{\mu \nu}=\partial_{\mu} A_{\nu}-\partial_{\nu} A_{\mu}-i\left(A_{\mu} \star A_{\nu}-A_{\nu} \star A_{\mu}\right)$.
The gauge fields in this theory do not commute, as the Yang-Mills term shows. Another unusual feature of this theory are complex, $\star$-gauge invariant Wilson loops [2]. Moreover a $\Theta$-deformed dispersion relation for the photon is expected 3 . Such a modification was observed for the NC $\lambda \phi^{4}$ model 4]. This is due to a remarkable feature of the NC field theories, the UV/IR mixing of divergences [5]. That property makes the perturbative treatment beyond one loop extremely difficult.

\section{LATTICE FORMULATION OF THE NC PLANE}

We carried out simulations involving two NC directions, as well as two commutative directions, which include the Euclidean time. To be explicit, we set $\Theta_{12}=-\Theta_{21}=\theta$, and $\Theta_{\mu \nu}=0$ otherwise. The parameter $\theta$ was assumed to be constant throughout the space-time.

In order to perform Monte Carlo simulations one needs to formulate the theory on a lattice. This is achieved by the operator relation [6]

$\exp \left(\frac{2 \pi i}{a} \hat{x}_{\mu}\right)=\hat{\mathbb{1}}, \quad \mu=1,2$,

which imposes a discrete non-commutative spacetime. After having obtained the lattice spacetime, it needs to be populated by U(1) link variables. The key to render their simulation - involving the non-local $\star$-product — feasible is the 
Morita equivalence [7] of $\mathrm{NC} \mathrm{U}(N)$ gauge theories to the twisted Eguchi-Kawai (TEK) model 8], with the action

$S_{\mathrm{TEK}}[U]=-\beta \sum_{\mu \neq \nu} \mathcal{Z}_{\mu \nu} \operatorname{Tr}\left(U_{\mu} U_{\nu} U_{\mu}^{\dagger} U_{\nu}^{\dagger}\right)$.

Here the $U_{\mu}$ are $\mathrm{U}(N)$ matrices. The twist $\mathcal{Z}_{\mu \nu}=$ $\mathcal{Z}_{\nu \mu}^{*}$ is given by $\exp \left(\frac{2 \pi i n_{\mu \nu}}{N}\right)$, with $n_{\mu \nu} \in \mathbb{Z}$. In our mapping we chose $n_{21}=-n_{12}=(N+1) / 2$ for some odd $N$, and $n_{\mu \nu}=0$ otherwise.

We discretized the commutative plane to a regular $(N-1) \times(N-1)$ lattice, where on each lattice site the NC plane is represented by a $N \times N$ TEK model ( $N$ is odd).

The non-commutativity parameter $\theta$ and the lattice parameters $N$ and $a$ are related as $[\underline{6}$

$\theta=\frac{1}{\pi} N a^{2}$.

In the $N \rightarrow \infty, a \rightarrow 0$ limit this theory describes a continuum gauge theory; which theory one obtains depends on the way this limit is taken. Keeping $\theta$ finite in the continuum and infinite volume corresponds to the double scaling limit. In the case of finite $N$ and $a$ the theory maps exactly to a $\mathrm{NC} \mathrm{U}(1)$ lattice theory $[6]$.

In order to be able to apply the heat bath algorithm, the action was linearized in $U_{\mu}$, as a generalization of the scheme of Ref. 9].

\section{RESULTS}

In the simulation results presented here we set $N=25$. The upper part of Fig. 11 shows the real part of the plaquette values at different $\beta$. In this case all six possible orientations of the plaquettes are averaged. The plot shows that the results match the anticipated asymptotic behavior 8 . The phase transition occurs at around $\beta \approx 0.35$.

The decay of Wilson loops observed from differently sized squares is shown in the lower part of Fig. 1 In that plot the results are split according to the plaquette orientations ( $\mathrm{NC}$, mixed and commutative). The real part of the NC Wilson loops oscillates, while eventually decaying towards zero. This is in qualitative agreement with the observations in Ref. 10 for $d=2$. For the

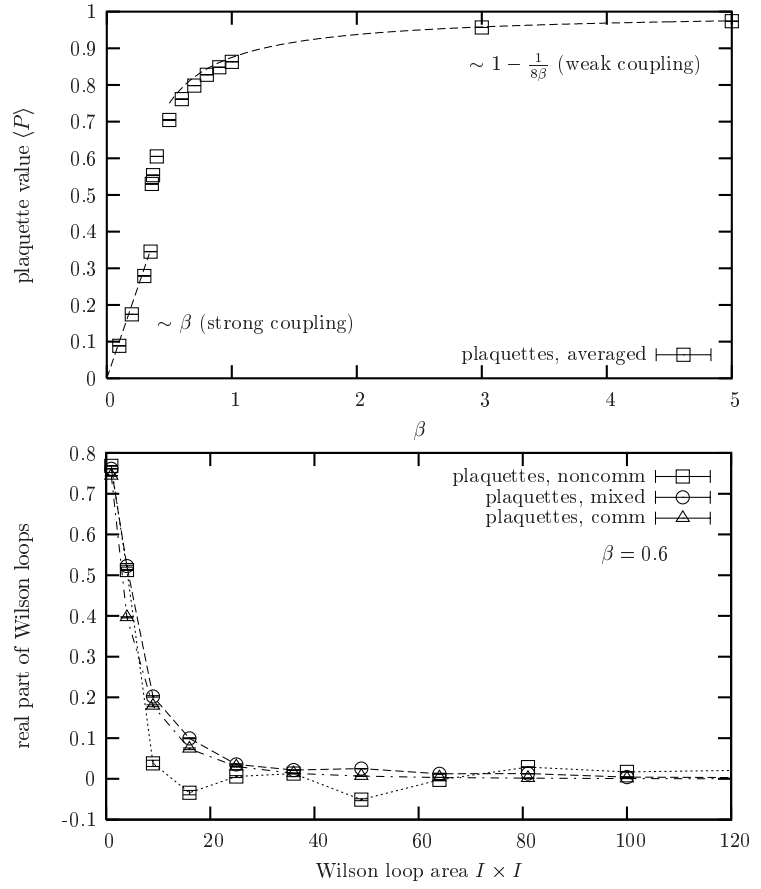

Figure 1. Above: The real part of the plaquettes, averaged over all possible orientations. The dashed lines show the asymptotic behavior expected based on a strong/weak coupling expansion. Below: The real part of the plaquettes, split according to their orientation.

other two types we see a monotonous decay towards zero. Notice that these results were obtained in the weak coupling phase.

We have calculated Creutz ratios $\chi$ for various rectangular Wilson loops. The results are shown in Fig. 22 From these we see that the string tension seems to approach zero as $\beta \rightarrow \infty$, which corresponds to the continuum limit.

Fig. [3 shows the correlation function of the plaquettes lying completely in the NC plane, and separated by $\Delta t$ in Euclidean time. These data were taken at $\beta=0.4$, so we are barely in the weak coupling phase. The decay seems to be exponential, but more statistics are required to confirm this behavior.

Fig. 4 shows the commutative analog to the correlation function discussed above. However, in that case the decay does not seem exponential. 


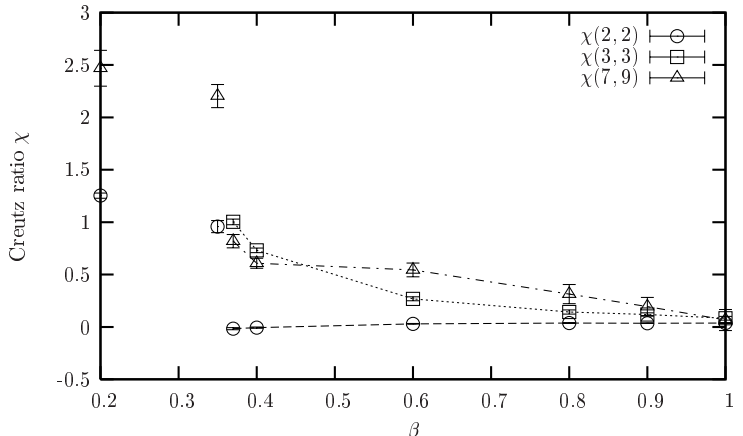

Figure 2. Different size Creutz ratios $\chi$ of rectangular Wilson loops, averaged over all planes, at various couplings.

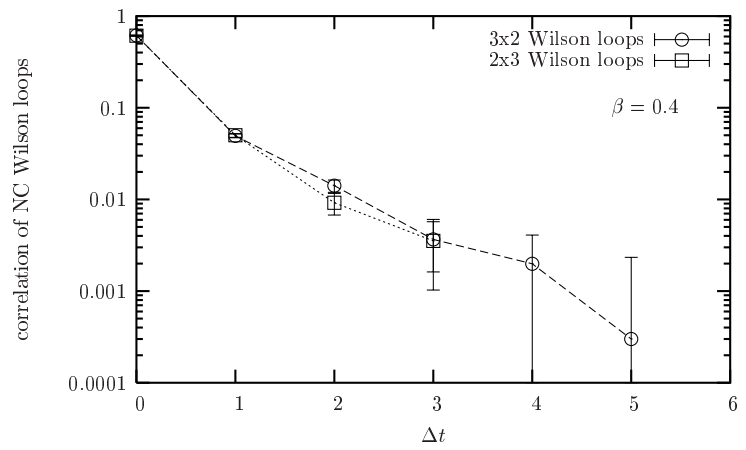

Figure 3. Temporal correlation of the plaquettes lying completely in the $N C$ plane.

\section{SUMMARY AND CONCLUSION}

We have simulated a $\mathrm{NC} \mathrm{U}(1)$ field theory. First observables such as the action, plaquettes, Wilson loops and Creutz ratios could be measured. The results for the action and plaquettes agree with asymptotic predictions. There seems to be a vanishing string tension at weak coupling in the part of the parameter space we explored.

Further work will hopefully allow us to identify a physical scale and to extrapolate to a NC continuum limit by means of double scaling (i.e. a fixed product $N \mathrm{C}^{2}$ ). Thus we hope to glimpse at the dispersion relations for the NC photon, which might allow us to confront the theory with nature.

Acknowledgement: We would like to thank the "Deutsche Forschungsgemeinschaft" (DFG) for generous support. The computations were performed on the IBM p690 clusters of

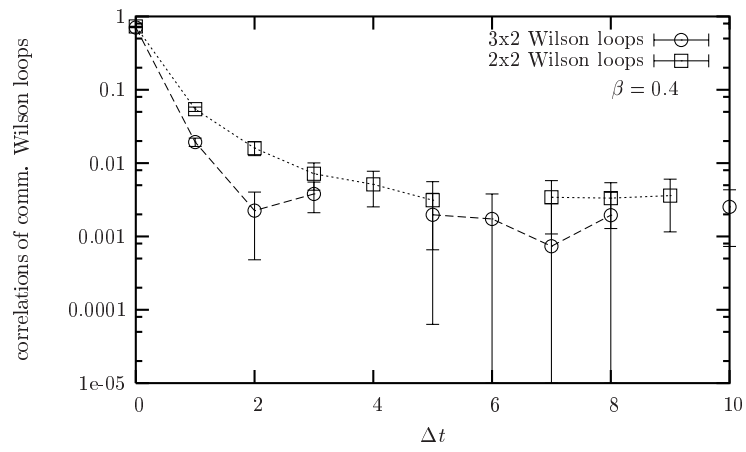

Figure 4. Temporal correlation of the plaquettes lying completely in the commutative plane.

the "Norddeutscher Verbund für Hoch- und Höchstleistungsrechnen" (HLRN).

\section{REFERENCES}

1. M.R. Douglas and N.A. Nekrasov, Rev. Mod. Phys. 73 (2001) 977. R.J. Szabo, Phys. Rep. 378 (2003) 207.

2. N. Ishibashi, S. Iso, H. Kawai and Y. Kitazawa, Nucl. Phys. B573 (2000) 573.

3. M. Hayakawa, hep-th/9912167. A. Matusis, L. Susskind and N. Toumbas, JHEP 12 (2000) 2. F. Ruiz Ruiz, Phys. Lett. B502 (2001) 274. K. Landsteiner, E. Lopez and M.H. Tytgat, JHEP 0106 (2001) 055.

4. S.S. Gubser and S.L. Sondhi, Nucl. Phys. B605 (2001) 395. G.-H. Chen and Y.-S. Wu, Nucl. Phys. B622 (2002) 189. P. Castorina and D. Zappalà, Phys. Rev. D68 (2003) 065008. W. Bietenholz, F. Hofheinz and J. Nishimura, JHEP 06 (2004) 42.

5. S. Minwalla, M. van Raamsdonk and N. Seiberg, JHEP 02 (2000) 020.

6. J. Ambjørn, Y.M. Makeenko, J. Nishimura and R.J. Szabo, JHEP 11 (1999) 29; Phys. Lett. B480 (2000) 399; JHEP 05 (2000) 023.

7. H. Aoki, N. Ishibashi, S. Iso, H. Kawai, Y. Kitazawa and T. Tada, Nucl. Phys. B565 (2000) 176.

8. A. González-Arroyo and M. Okawa, Phys. Rev. 27D (1983) 2397.

9. K. Fabricius and O. Haan, Phys. Lett. 143B (1984) 459.

10. W. Bietenholz, F. Hofheinz and J. Nishimura, JHEP 09 (2002) 9. 